\title{
THE EFFECTS OF PC-BASED TRAINING ON NOVICE DRIVERS' RISK AWARENESS IN A DRIVING SIMULATOR
}

\author{
Anuj K. Pradhan ${ }^{1}$, Donald L. Fisher ${ }^{1}$, Alexander Pollatsek ${ }^{2}$ \\ ${ }^{1}$ Department of Mechanical and Industrial Engineering \\ ${ }^{2}$ Department of Psychology \\ University of Massachusetts \\ Amherst, Massachusetts, USA \\ E-mail: apradhan@ecs.umass.edu \\ E-mail: fisher@ecs.umass.edu \\ E-mail: pollatsek@psych.umass.edu
}

\begin{abstract}
Summary: Novice drivers are almost nine times more likely to die in a crash than more experienced drivers. This increased risk has been found to be largely due to novice drivers' inability to predict the risks in the roadway ahead. A PC-based Risk Awareness and Perception Training Program (RAPT) was developed to teach novice drivers about different categories of risky situations likely to be encountered while driving. The format was an interactive multimedia presentation with both plan (i.e., top down) views and perspective views of roadway geometry that illustrated generally risky scenarios along with information about the type of risks and the relevant areas that attention should be allocated to in order to detect the risks. A set of novice drivers was trained with this program. The eyemovements of the participants were then evaluated in a driving simulator to determine whether areas of potential risk were fixated, and their performance was compared to a separate set of untrained novice drivers. The ability of the novice drivers to identify risks in static views improved after they completed the training program. More importantly, the trained novice drivers were significantly more likely to correctly fixate on risk relevant areas in the simulated driving environment than the untrained drivers 3-5 days after training.
\end{abstract}

\section{INTRODUCTION}

Younger novice drivers (i.e., sixteen year old drivers with six months or less of experience) are involved in the highest number of fatal crashes per 100 million vehicle miles driven. The accident rate for this group is 9.3 fatal crashes per 100 million vehicle miles as compared to 1.4 for drivers between 45-54 years of age (Insurance Institute for Highway Safety, 2003). The National Highway Traffic Safety Administration ascribed this over-representation to the inexperience, risk-taking propensity and immaturity of the novice drivers (NHTSA, 1994). Newer studies, however, indicate that the novice drivers' lack of experience is the major cause of crashes. For example, researchers analyzed close to 1000 crashes involving novice drivers (McKnight \& McKnight, 2003) and concluded that inexperience was the major factor in the crashes (42.7\% of the crashes). The behaviors classified as inexperience in the above-mentioned study included failure to search appropriately in areas of the roadway - either those ahead, to the sides, or to the rear. Treat et al. (1979) reported similar findings where visual search, speed adjustment and attention were responsible for driver crashes and Gregerson (1996) estimated that up to $70 \%$ of the novice drivers' errors were attributable to driver inexperience. 
Driving simulator studies comparing novice drivers with experienced drivers also indicate that driver inexperience is a major cause in differences in driver behavior (Fisher et al., 2002; Pradhan et al., 2004, in press). In Pradhan et al. (in press), the eye movements of younger novice drivers were compared to those of younger experienced drivers (19-29 yrs) and older experienced drivers (60 years and older). The locations of eye fixations were recorded for the participants as they drove through risky scenarios in a simulator. Overall, the novice drivers engaged in behaviors indicative of risk recognition $35.1 \%$ of the time, compared to $50.3 \%$ and $66.2 \%$ for the younger experienced drivers and the older experienced drivers respectively.

Effective training for improving risk awareness skills should be able to reduce the effects of this lack of experience in novice drivers. However, standard driver training programs do not seem to be giving specific risk awareness training to the novice drivers in their curricula, as shown by the fact that the novice driver fatality rate has been increasing as compared to the decreasing fatality rates of more experienced drivers. There were 19 driver deaths for every 100,000 licensed 16year old drivers in 1975, and this increased to 35 deaths per 100,000 in 1996 (Insurance Institute for Highway Safety, 1998). In contrast, for the overall driver population, the death rate has decreased from 15 deaths per 100,000 licensed drivers in 1975 to 12 driver deaths per 100,000 in 1996. The driver education curriculum could be supplemented with PC based risk awareness training programs. With today's technology, a PC based program, distributed on CD-ROMs or made available on the Internet, would be an appropriate media for delivering the training.

A number of PC-based training programs have already been developed to address the problem of training novice drivers for risk awareness. Some of these programs have been evaluated [e.g. DriverZED, developed by AAA Foundation for Traffic Safety and evaluated by Fisher et al., 2002; DriveSmart, developed and evaluated by the University of Monash Accident Research Center, Regan, Triggs, \& Godley, 2000, and DATS (Driver Assessment and Training System), developed and evaluated by Systems Technology, Inc., Allen et al., 2003]. These evaluations have shown that PC-Based training programs help novice drivers perform better in a simulated environment. The Risk Awareness and Perception Training Program (RAPT) described in this paper is the second-generation version of the training program described by Fisher et al. (2004). This program is an interactive multimedia presentation of ten risky scenarios covering three broad categories of risk. The program can be distributed on CD-ROM media or downloaded from the Internet. In the evaluation of the first generation version of RAPT, the trained participants were tested on the driving simulator immediately after training. Moreover, in the first generation version, as in the second generation version, the views of scenarios that drivers see on the PC are mostly plan views that are very different in format from their simulated drives through a virtual world. Thus, the fact that the trained drivers performed better on the driving simulator was of real significance, even though performance on the simulator was evaluated immediately after PC training with the first generation version. Still, one would like to know whether the effects of training persisted over a time frame of just one hour. In this experiment, the participants trained with the second generation version (RAPT) were evaluated on the driving simulator an average of 3.9 days after the initial RAPT training. 


\section{EXPERIMENT}

\section{PC Training}

The training program had ten risky scenarios spread across three categories: 1) Obstruction (a vehicle obscured the participant driver's view of a risk, e.g., a stopped truck obscured a driver's view of a pedestrian crossing in front of the truck); 2) Sign Ahead (e.g., a Stop Sign Ahead sign warned drivers of a stop sign which itself was largely obscured by vegetation until the last minute); and 3) Visible Pedestrian/Vehicle (e.g., a vehicle immediately ahead of the driver and turning to the right or left might stop suddenly to avoid hitting a pedestrian crossing the street perpendicular to the participant driver's path of travel). All scenarios were displayed in a topdown view. Additionally, in some cases, snapshots of actual driving scenes were displayed as perspective views to illustrate a scenario more clearly. The participant was instructed to drag red circles or yellow ovals over the schematic of the scenario (using the mouse). The red circles were to be dragged over to the area of the scenario that should be monitored more or less continuously by a driver and the yellow ovals were to be moved to areas on the scenario that contained a vehicle, pedestrian, or sign that would be hidden from the driver at the car's location in the plan view but which could have relevance for the driver's path of travel subsequently (Figure 1a). Additionally, for each scenario, the participants were asked 2-3 questions relevant to the scenario and the risks inherent in it.

Participants. Twelve drivers (six males and six females) were recruited from local area driving schools to undergo the risk awareness training. The drivers all had Learner's Permits. The mean age of this participant group was 16.72 years.

Experimental design and procedure. The training program (RAPT) had five sections; Instruction, Pre-test, Training, Questions and Post-test. (1) The Instruction section served to familiarize the user with the program interface and layout. Three practice sessions were included in the instruction phase that were used both to illustrate the top-down view in relation to the regular perspective views and for practice in dragging and dropping the yellow ovals and red circles. The user was also familiarized with answering questions in the provided text boxes. (2) The Pre-test section presented the ten scenarios and the user was expected to drag the red circles and yellow ovals over to the relevant areas. There was no feedback provided to the participants in this phase about their responses. (3) In the Training section the participant was shown three or four different types of screens per scenario. In the first screen, the Subject Response Screen (Figure 1a, without the red circles or yellow ovals positioned in the correct location), the participant was shown the plan view of the scenario with one or more vehicles and/or pedestrians along with three red circles and three yellow ovals to the side. The participant was asked to drag the red circle and yellow ovals onto the relevant areas on the screen. The participant was then shown the Vision Obstruction Screen (Figure 1b) that indicated the areas of the roadway that were obstructed from the driver's view and provided explanations of the various risks that could arise in the scenario due to the hidden elements. Finally, the participant was shown the Answer Explanation Screen that indicated the correct locations of the yellow ovals and the red circles along with explanations for why they were placed in those locations. For some scenarios, the participant was shown an additional Visualization screen (Figure 1c) that had a perspective view along with the plan view of the scenario to illustrate more clearly the scenario and to aid in the visualization of the scenario. (4) The Question section presented the ten scenarios to the 
participant with questions about the risks in the scenario. Feedback was given to the participant after each scenario's questions had been input. (5) Finally the Post-Test section presented the plan views of the scenarios to the participants again and they were again instructed to move the red circles and yellow ovals to relevant areas. The performance of the participants in this section was compared to their performance in the Pre-Test section.

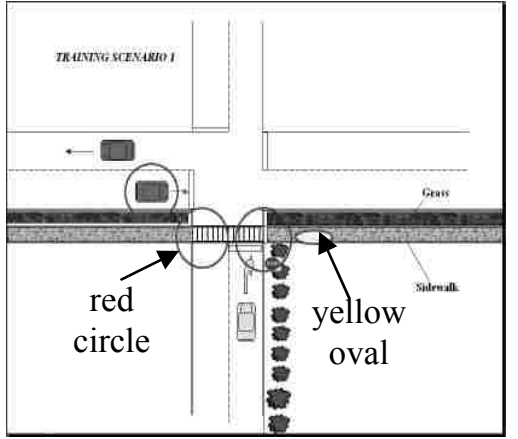

a) Subject Response Screen

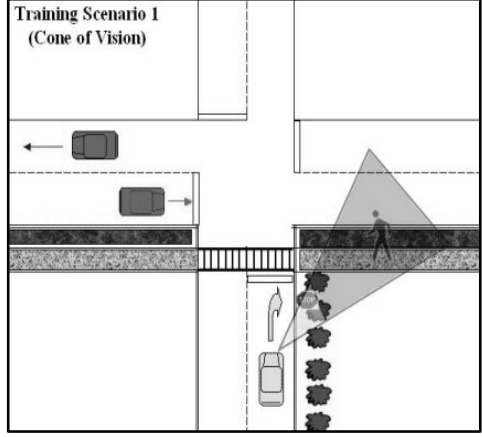

b) Vision Obstruction Screen

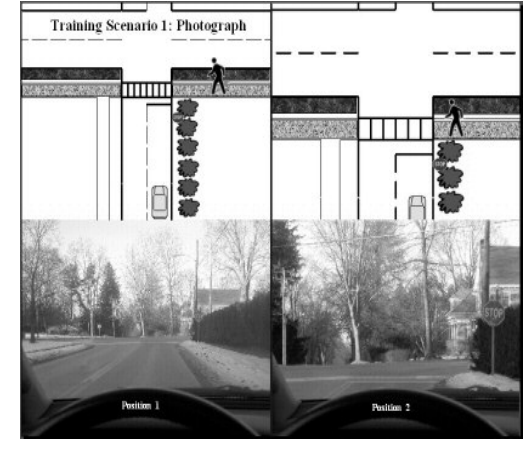

c) Visualization Screen

Figure 1. PC Training Program

The PC-based training took about 90 minutes to complete. After the PC training, the participants returned after 3-5 days for the second part of the experiment, the simulator testing.

\section{Simulator Testing}

To test for the effects of the PC training program, the participants were evaluated in a driving simulator to see if they could generalize the learning from a plan view scene to a more realistic environment. The trained group of participants drove through a set of sixteen virtual scenarios in a fixed base advanced driving simulator. The scenarios included all of the ten scenarios in the PC training program and an additional set of six scenarios. The latter, which were significantly different from the trained scenarios, were included to determine if the training would generalize beyond the trained scenarios. A set of matched, untrained, control participants was also tested on the simulator. The participants' fixation locations were recorded and converted to external points of gaze that were superimposed on the driving scene.

Participants. The twelve participants who had completed the PC-based training program were evaluated in the simulator as the experimental group 3-5 days after PC training. Another set of twelve participants were evaluated on the simulator as the control group without having been trained on the PC training program. All members of the control group had their Learner's Permit. The control group of six males and six females had a mean age of 16.44 years.

Equipment. An advanced fixed-base driving simulator was used for the evaluation. The simulator includes a fully equipped 1995 Saturn sedan with three screens located in front of it. The screens display 135 degrees of horizontal view. The virtual world is displayed on each screen at a resolution of $1024 \times 768$ pixels at a frequency of $60 \mathrm{~Hz}$. The participant sits in the car and operates the controls as one would in the real world, moving through the virtual world according to his or her inputs. The simulator is equipped with an integrated eye and head tracking system 
(ASL 5000). The participant was fitted with the eye-tracker during the simulation. The eyetracker recorded the eye-movements of the participant at $60 \mathrm{~Hz}$ and converted it to point of gaze information. This point of gaze was superimposed as crosshairs onto a real time video of the drive indicating the position of the driver's gaze in the visual field at each point in time.

Experimental design and procedure. The sixteen scenarios for the driving simulation consisted of the ten scenarios that were used for the PC Training and six additional scenarios that did not resemble the PC Training Scenarios. The six additional scenarios could also be separated into the three categories mentioned earlier: Obstruction, Sign Ahead and Visible Pedestrian/Vehicle. The sixteen scenarios were presented to the participants in four separate drives, with each drive containing four embedded scenarios (Figure 2). The participants were calibrated on the eyetracker and then were given a practice drive to familiarize them with the simulator controls. The participants were free to drive through the practice block until they were comfortable with the steering, braking and other controls of the car.

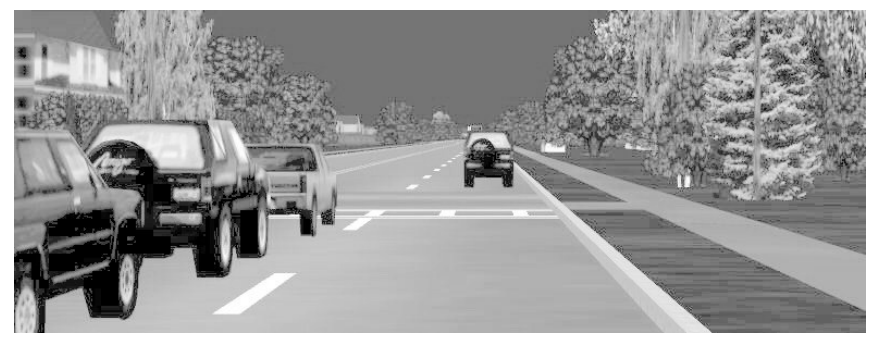

Figure 2. Simulator Scenario

\section{RESULTS AND DISCUSSION}

\section{PC training}

For each of the three categories, the participants were better able to identify, after training, the areas in a scenario that should be monitored closely and areas that could have hidden risks as indicated by the placement of red circles and yellow ovals respectively (see Figure 3 ). The improvement in test scores was significant for both the red circles, $39 \%$ to $67 \%, t(11)=4.49, p$ $<0.001$, and the yellow ovals, $51 \%$ to $79 \%, t(11)=5.25, p<0.001$.
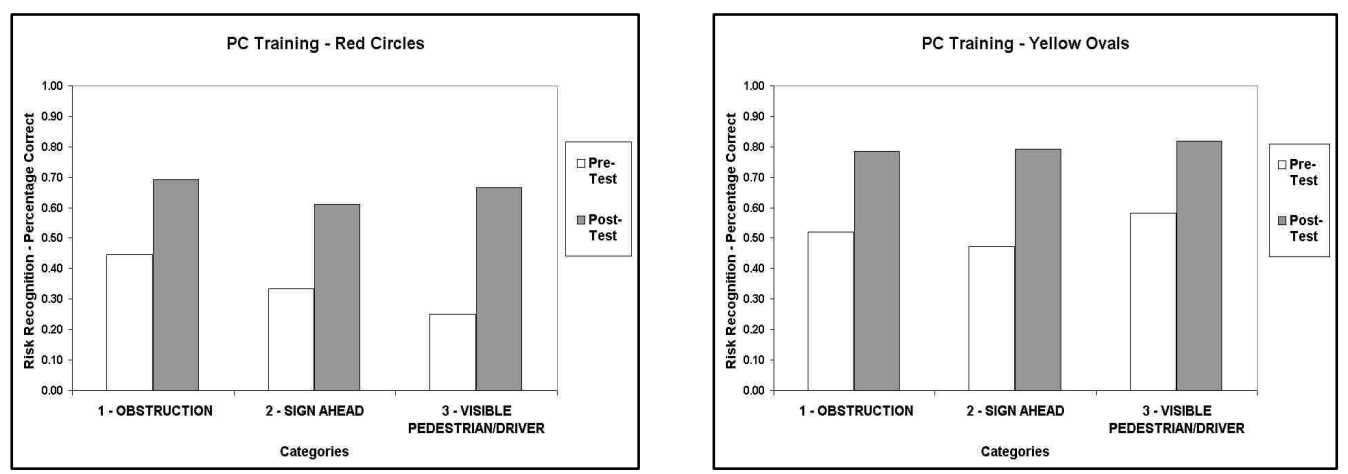

Figure 3. PC Training Results 


\section{Simulator evaluation}

Although the improvements above were significant, the crucial question was whether the training on the PC program would lead to improvement in a more realistic environment. For this, the eye gaze data was analyzed for fixations on risky areas of the scene. The novice drivers who had the advantage of the PC-based training performed better than the untrained group, fixating on the risky areas $48.6 \%$ of the time, compared to $30.7 \%$ for the untrained drivers. This difference was significant, $t(22)=2.24, p<0.05$. Figure 4 shows the difference in the fixation percentages for the trained and untrained drivers across three categories. Furthermore, the difference in performance between the trained and untrained drivers for the six test scenarios that were not in the training session was virtually identical to that for the ten scenarios that had been in the training session.

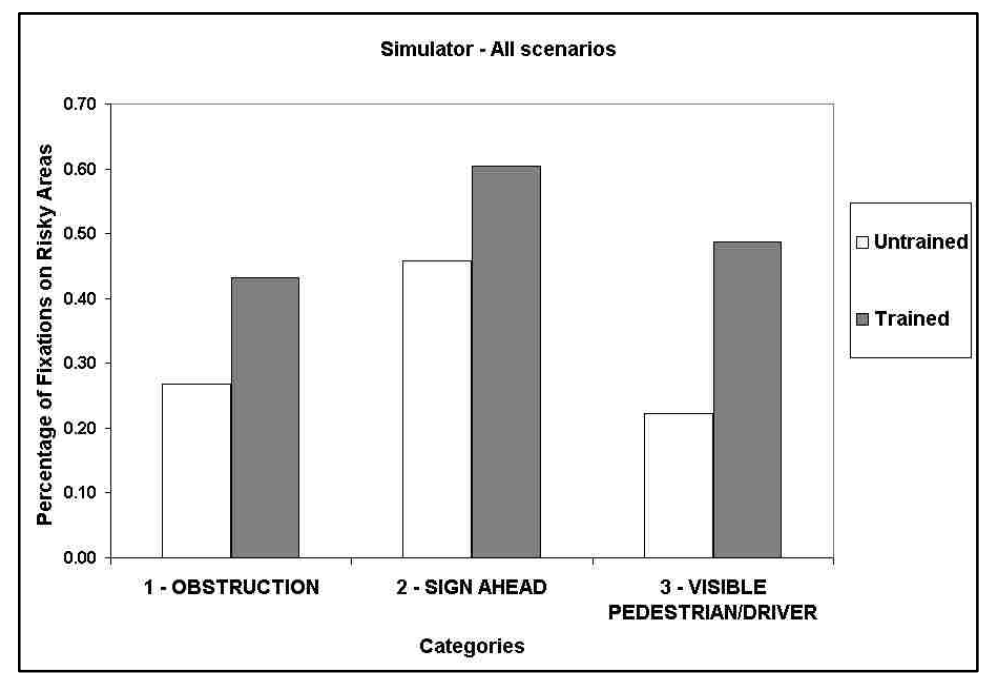

Figure 4. Simulator Results

Overall, the training was shown to be effective. Participants were significantly better at identifying the critical risky areas in the RAPT post-test than in the RAPT pre-test. Of greater importance was the test in the driving simulator. The trained drivers were performing about as well as more experienced drivers in prior studies and were almost $40 \%$ more likely to fixate risk relevant areas than the untrained drivers. This is a relatively large increase given that the PC trained drivers saw primarily overhead views on the PC, views which differed greatly from the perspective views that they saw on the driving simulator. Of equal importance was that the effects of training lasted over a span of several days between PC training and simulator test and the effects were about as large as in a prior study (Fisher et al., 2004) in which the simulator test was administered right after the training session. This offers some real promise that the increases in risk awareness might transfer to the open road if the interval between training and driving is relatively short. Perhaps such a short window is sufficient. Behaviors that are learned on the PC may turn into habits if they become part of the novice driver's routine when first on the open road. 


\section{REFERENCES}

Allen, R. W., Rosenthal, T. J., Park, G., Cook, M., Fiorentino, D., Viirre, E. (June 2003) Experience with low cost, PC based system for younger driver training (paper no. 614). Hawthorne, California: Systems Technology, Inc.

Fisher, D. L., Laurie, N. E., Glaser, R., Connerney, K., Pollatsek, A., Duffy, S. A. and Brock, J. (2002). The use of an advanced driving simulator to evaluate the effects of training and experience on drivers' behavior in risky traffic scenarios. Human Factors, 44, 287-302.

Fisher, D. L., Narayanaan, V., Pradhan, A. and Pollatsek, A. (2004). The Use of Eye Movements to Evaluate the Effect of PC-Based Risk Awareness Training on an Advanced Driving Simulator. Proceedings of the Human Factors and Ergonomics Society's Annual Meeting, New Orleans. In press.

Gregersen, N. P. (1996). Young car drivers: Why are they over represented in car accidents? How can driver training improve their situation? (VTI Rapport 409A). Linkoping: Swedish National Road and Transport Institute.

Insurance Institute for Highway Safety. (1988). Sixteen-year-old drivers' death rates rising at alarming rate. Status Report, 33 (6), 1-2.

Insurance Institute for Highway Safety (2003). Fatality Facts, Older People. http://www.highwaysafety.org/safety_facts/fatality_facts/olderpeople.htm (Accessed April 10, 2005).

McKnight, J. A. and McKnight, S. A. (2003). Young Novice Drivers: Careless or Clueless. Accident Analysis and Prevention, 35, 921-925

National Highway Traffic Safety Administration. (1994). Research agenda for an improved novice driver education program (Report to Congress, May 31, 1994). Washington, DC.: Department of Transportation.

Pradhan, A. K., Hammel, K. R., DeRamus, R., Pollatsek, A., Noyce, D. A. and Fisher, D. L (2004). The Use of Eye Movements to Evaluate the Effects of Driver Age on Risk Perception in an Advanced Driving Simulator. Human Factors. In Press.

Regan, M. A., Triggs, T. J. and Godley, S. T. (2000). Simulator-based evaluation of the DriveSmart novice driver CD-ROM training product. Proceedings of the Road Safety Research, Policing and Education Conference.

Treat, J.R., Tumbas, N.S., McDonald, S.T., Shinar, D., Hume, R.D., Mayer, R.E., Stansifer, R.L., Castellan, N.J., 1979. Tri-level study of the causes of traffic accidents (Final Report). In: Causal Factor Tabulations and Assessments, vol. I. Bloomington, IN: Indiana University Institute for Research in Public Safety. 